\title{
Improving representation in physical sciences using a departmental action team
}

\author{
Katherine Rainey, ${ }^{1}$ Joel C. Corbo, ${ }^{2}$ Daniel Reinholz, ${ }^{2}$ and Meredith Betterton ${ }^{1}$ \\ ${ }^{1}$ Department of Physics, University of Colorado, Boulder, CO 80309 \\ ${ }^{2}$ Center for STEM Learning, University of Colorado, Boulder, CO 80309
}

\begin{abstract}
Women and minority students are largely underrepresented in the physical sciences. Here we describe a faculty-, student-, and staff-led group, called a Departmental Action Team (DAT), created to study the recruitment and retention of women and underrepresented minorities in one physical sciences department. As a result of the DAT's work, the department has created a new standing committee to improve outreach and support for students from underrepresented groups. We describe how the efforts of the DAT created a departmental context that was able to leverage a campus-level diversity initiative to support meaningful, department-wide discussions and a plan for improving diversity and equity in the major. As such, we present an example of departmental change that could serve as a model for other physical science departments seeking to create similar change.
\end{abstract}

\section{INTRODUCTION}

The lack of representation of women, underrepresented minorities (URMs), and people at the intersections of these groups in the physical sciences is an issue of ongoing concern. For instance, in fields such as computer science, physics, and engineering, only about $20 \%$ of undergraduate degrees are awarded to women and $15 \%$ are awarded to URM students [1]. This paper focuses on one effort to address this issue, through the Inclusion Committee (IC) that was formed in a physical sciences department at a large research university; we refer to this department as Potions to preserve confidentiality. The IC aims to improve the retention, recruitment, and support of traditionally underrepresented students.

This paper tells the story of how grassroots efforts can lay the foundation for meaningful department change. While the IC was initially faculty-driven (with one postdoc), it grew over time to include students and staff. Moreover, we argue that the presence of such grassroots efforts can pave the way for more productive interactions with university administration. We highlight this potential by describing the top-down Diversity Initiative (DI) that was mandated by the university administration, and how IC was able to respond to it. We conclude the paper by describing key features of the IC, to guide others in their local change efforts.

\section{THEORETICAL BACKGROUND}

There is growing recognition that improving undergraduate STEM education requires more than spreading teaching techniques; instead, it requires attention to scaling and sustaining systemic changes [2]. A university is a complex, multifaceted system, and for change to succeed, it must operate at multiple levels. Nevertheless, there is now growing support that the department itself is a critical locus for change, as it operates as a relatively coherent unit on a campus [3].

The STEM Institutional Transformation Action Research (SITAR) project is a holistic institutional change effort that has responded to the above calls for systemic change. The SITAR framework for change posits that change efforts should focus on faculty, departments, and administration and that ignoring any of these levels would likely inhibit the effectiveness of an effort [4]. One proposed model for change focuses on a combination of grassroots faculty efforts (bottomup) and administrative initiatives (top-down) that coordinate with one another to result in departmental change [5].

In the SITAR project, the primary mechanism for supporting grassroots efforts was through Departmental Action Teams (DATs). DATs are externally-facilitated groups that consist of faculty, staff, and students within a single department working on a broad-scale educational issue of departmental importance. DATs have several key features:

External facilitation: DATs are facilitated by postdocs from outside the department who have expertise in educational research and organizational change that they can use to support the DAT's efforts. The facilitators handle the logistics of organizing the DAT, provide an outside perspective that pushes DAT participants to question their assumptions, connect the DAT to other campus and national resources, and deliberately strive to create a culture of collaboration and inclusion within the DAT.

Participant agency: DAT participants choose the issue that their DAT will address and the approach to their work. The facilitators support them in making these decisions by recommending courses of action, but they do not impose an agenda from the outside.

Cross-cutting focus: DATs focus on issues of departmental importance (e.g., underrepresentation of certain groups among undergraduates or coherence of a department's curriculum) rather than issues that impact a single course. This supports the a DAT's goal of making changes that scale to an entire department.

Outcomes-driven: The first meetings of a new DAT are devoted to creating a shared vision among the DAT participants of what they want to achieve. This vision guides the work of the DAT: ideally, the DAT's actions or decisions flow from the vision rather than from the personal preferences of any individual participant.

Evidence-based decisions: DAT participants spend a significant time gathering and analyzing information to better understand the issue they are trying to address. In this way, 
their decisions can be based on evidence rather than their own idiosyncratic understanding of their department.

Although the SITAR change model pairs bottom-up and top-down efforts, there is no guarantee that the changes intended by faculty will be consistent with administrative mandates. Thus, grassroots efforts must be flexible, so that they can adapt and respond to the administration in a meaningful way. Below, we describe how the structure of the IC allowed it to respond to the top-down Diversity Initiative (DI).

\section{DEVELOPMENT OF THE IC}

The IC began in Fall 2014 as a faculty effort facilitated as a DAT through SITAR. In May 2015, the effort was formally recognized as an ad hoc committee by the Potions Department. In May 2016, the IC became recognized as a permanent standing committee by unanimous vote of the faculty. In transitioning to a committee, the members of IC maintained the features of a DAT discussed in Sec. II. In this section, we discuss some of the development and accomplishments of the IC to illustrate the importance of maintaining these features and to contextualize our recommendations in Sec. IV.

\section{A. Origins as a DAT}

The Potions DAT was formed in the Fall of 2014, and the details of its first year are discussed elsewhere [6]. We briefly recount aspects of that year relevant to the rest of our discussion in this paper.

During its first year, the Potions DAT consisted of five participants: one post-doc (the director of a departmental outreach program), two pre-tenure faculty members, and two tenured faculty members. The DAT met for an hour every other week for the 2014-2015 academic year, facilitated by two SITAR postdocs. One of its main activities was to collect and analyze data about the attrition and representation of Potions undergraduates, which allowed it to quantify the department's weak points in recruitment and retention of women and URM students. For example, women were far more likely to drop the introductory Potions course than men, even when accounting for declared major, and women and URM students who expressed interest in Potions on their university applications and were admitted were less likely to matriculate than their male and white peers. The DAT compiled its findings into a report and disseminated it via email to all faculty, students, and staff in the department.

\section{B. Diversification of Membership}

When the IC convened in the fall of 2016, it had four faculty members appointed to it by the Potions department chair: three of the original faculty DAT participants (at their request) and one new faculty member. Additionally, the two SITAR postdocs agreed to facilitate the group. As the IC started its work, its members collectively recognized the need to include members with other roles in the department. In this sense, this diversity-focused group recognized the lack of diversity in its own membership and how that would inhibit its goals of making the department more inclusive.

By the end of the fall semester, the group had expanded to include two staff members, three graduate students, and two undergraduate students. The graduate students were recruited via email from the entire Potions graduate student population, and the undergraduate and staff participants were personally asked to join by existing IC members. The IC is unique in terms of departmental committees, because it contains so many non-faculty members; rarely do faculty, staff, postdocs, and students all work together on issues of common interest and concern (in the Potions department or elsewhere).

Despite concerns from some IC members that such a large increase in membership would make the IC unmanageably big, in practice the group's ability to get work done was enhanced because of the diversity of its membership. Inclusion of multiple levels within the department allowed more voices to be heard, which allowed the group to make better decisions based on, e.g., the lived experiences of undergraduates rather than faculty guesses about their experiences. Because the DAT had already created a culture of mutual respect that carried into the IC, these voices were recognized as valuable rather than dismissed. Additionally, more participants allowed for a larger number of tasks to be executed by the IC at one time, increasing the productivity of the committee. Rather than getting in each other's way, new members were able to work synergistically because they could all align their interests with the shared vision that the group began to develop during its time as a DAT.

Finally, the IC diversified not only in terms of academic level, but also in terms of other demographics. The IC is currently majority female, has multiple representatives from the LGBTQ+ community, and includes students from nontraditional academic backgrounds. Nevertheless, due to the overall demographics of the Potions department, the IC is primarily white.

\section{Accomplishments}

Here we present accomplishments of IC, focusing on a university diversity initiative, to illustrate the committee's productivity.

\section{The Diversity Initiative (DI)}

In the spring of 2016, a Diversity Initiative (DI) was announced by the administration of Potion's university. The DI required that all departments create a document that addressed the following questions: 
1. How does your department define excellence with respect to the activities that it carries out, and what values and behaviors are associated with excellence?

2. How do the efforts within the department lead to inclusion of all students in achieving academic success, student social development, and enhancing the reputation of the department and the university?

3. Given the department's definition of excellence and inclusion, how will the department attain inclusive excellence?

Despite issuing this mandate, the administration provided departments with no support to achieve it. As a result, several departments ignored this initiative altogether. In the Potions department, the chair asked the IC if it could organize the department's response to the administration. Other than the IC, there were limited mechanisms within Potions to respond to the DI. As such, the IC had created fertile ground for responding to the DI in a meaningful way.

Consistent with SITAR's model for change (see Sec. II), the IC had a flexible structure that allowed it to respond to the administrative call. Rather than simply generating a short document to answer the administration's three questions, the IC leveraged the DI as an opportunity to have departmentwide conversations about diversity and inclusion. Accordingly, the IC scheduled five "town hall" meetings, one each for faculty, staff, postdocs, graduate students, and undergraduate students. As much as possible, the meetings were facilitated by IC members of the appropriate group (i.e., undergraduates facilitated the undergraduate meeting). This was done in an attempt to limit status differences between the facilitators and participants that might inhibit the participants from being honest, which is another benefit of having crosssections of the department represented on the IC. Through a series of activities, participants at each meeting were able to articulate their criteria for being an excellent member of the Potions department, share stories of inclusion and exclusion they have experienced in the department, and brainstorm ideas for how to make the department more inclusive. The IC compiled these responses into one cohesive document that represents the views of all five groups; this document was used as the Potions department's contribution to DI.

In this way, the Potions department was able to meet the requirements of the administration, but in addition to this external use, the meetings and information gathered by the IC were also valuable to the committee and the department itself. First, many participants at the meetings stated that they were happy that they had a space within the department to discuss issues of inclusion; this in and of itself is a small step towards changing departmental culture around inclusion. Second, the meetings raised the visibility of the IC among all members of the department, providing IC more legitimacy. Finally, the ideas generated at the meetings, especially with respect to ways to increase inclusion, will inform the actions of the IC moving forward. Thus, the IC leveraged the administration's call to support its own work. This illustrates how work at faculty and administrative levels together can be used to influence a department.

\section{Other Activities}

In addition to the DI, the IC also initiated a number of other activities during its first year. These included:

Contacting admitted students: As a result of analyzing admissions data, IC decided to initiate a new recruitment activity. In partnership with two other Potions organization, the IC organized a calling session in which current Potions students contacted all female and URM admitted Potions students, with personalized follow-up emails from Potions faculty. During this process, 176 students were contacted and provided with at least two Potions department contacts to whom they could send any questions.

Strategic relations: The IC has partnered with the university's strategic relations (SR) office to improve advertising for the department. SR will work with the IC to redesign the department's website and other promotional materials to better reflect the opportunities the department provides for women and URM students (to encourage more of these students to matriculate) and to make information about navigating the university easier to access (to support current students, especially those who are non-traditional or who are the first in their family to attend college).

Gender-neutral bathrooms: The IC has begun to work with the Potions building manager to do a survey of the bathrooms in the building in order to designate some bathrooms as gender-neutral. This is a first step in broadening the scope of the IC to include LGBTQ+ students in addition to women and URM students.

\section{Future Directions}

Quantitative measures of the IC's success are not yet available since it is only in its second year of work. Nevertheless, the departmental response to the IC's efforts has been largely positive. The committee plans to maintain its current structure, including membership by non-students, although this will depend on which faculty are assigned by the chair to the committee. Moreover, the IC plans to focus its efforts moving forward on positively impacting departmental culture and fostering community, as well as continuing to work on advertising and recruitment strategies.

The IC has planned several activities to address community and culture within the Potions department. During the DI meetings, undergraduate students expressed a desire for more opportunities to meet other Potions majors. In response, the coming academic year will begin with an undergraduate welcome event hosted by the IC. This event will provide space for new and returning Potions majors to meet each other and socialize, which is difficult within a large department. As another response to the DI, monthly meetings open to all Potions department members will be held to maintain the con- 
versations started during the DI process. While the final form of these meetings has not been determined, they will likely involve readings and discussions of inclusion topics relevant to the department, and one of their goals will be to normalize conversations about inclusion among all members of the department. These meetings will be prefaced by a "State of Inclusion" event, in which the IC will update the department on its efforts, solicit volunteers to support future IC events, and build community. Additionally, the IC plans to augment the current Potions undergraduate advising process by partnering with a student-led mentoring program to provide their undergraduate mentees with faculty mentors.

At the end of year two, the formal support of the IC through SITAR ended. Nevertheless, the IC members have unanimously agreed to continue operating the IC using the same principles as the DAT.

\section{DISCUSSION AND RECOMMENDATIONS}

The accomplishments of the IC in its first two years are a significant change from previous department efforts to address representation and inclusion. These achievements were made possible by the unique structure and organization of the IC, which we discuss here. We recommend that these factors be taken into consideration if another department or institution tries to create a departmental committee with a DAT-like structure.

Facilitation: The IC benefitted greatly from having facilitators to guide its meetings. The facilitators created agendas to guide the hour-long meetings, which allowed for efficient discussion and planning. Additionally, the facilitators kept thorough meeting notes so all committee members were updated on progress and plans, even if they missed a meeting. Between the bi-weekly meetings, facilitators reminded subgroups working on specific projects to be ready to present their progress at the next whole-committee meeting.

Goals and vision: When the Potions DAT was first created, the IC discussed broad goals, including drafting a vision of how they wanted the department to be. This vision consisted of smaller goals which had associated action items. Achieving these action items were "small wins" that kept the group motivated throughout their first two years. Additionally, goals and activities were regularly revisited to ensure their mutual alignment. Moreover, the focus on goals, rather than specific solutions, allowed the IC to be open-minded in responding to the top-down DI, so it could leverage this administrative call to further its own work.

Multi-level: Because the IC addresses issues at multiple levels of the department, people from each academic level are members of IC. This provided faculty with perspectives that would not be available if they were working only with other faculty, like most departmental committees. Having student input was helpful in deciding what activities would be most beneficial for improving student experiences and lead to better recruitment and retention of underrepresented students.

Participant Agency: IC was formed out of a desire to improve recruitment, retention, and representation of students in Potions by faculty who were interested in the issue. Additional members shared the same concern. This voluntary, self-selected engagement in the group made it productive because everyone in the group cared about the work they were doing. This commitment was an important motivator for IC members to spend extra time outside of meetings to work on IC-related projects in addition to their other responsibilities. This allowed for multiple tasks to be accomplished in a relatively short amount of time.

\section{CONCLUSION}

Change is complex; to be successful, it requires cooperation from a large number of stakeholders with various interests. This short case study illustrates how a departmental committee can make progress on addressing a complex issue by DAT-like principles. It also illustrates how departmental efforts can be designed to take advantage of top-down opportunities when they arise, despite the often-perceived mismatch between the priorities of a university's administration and its faculty. We hope that this example will encourage others to rethink how their department goes about trying to increase inclusion (and address other complex problems).

\section{ACKNOWLEDGMENTS}

We thank the Association of American Universities and the Helmsley Charitable Trust for funding SITAR. We also thank members of the IC for the time and energy they put into their work and the Potions department for supporting this effort.
[1] National Science Foundation, Tech. Rep., Washington, DC (2013).

[2] President's Council of Advisors on Science and Technology, Tech. Rep., Washington, D.C. (2012).

[3] Association of American Colleges and Universities, Tech. Rep., Washington, DC (2014).

[4] J. C. Corbo, D. L. Reinholz, M. H. Dancy, S. Deetz, and N. Finkelstein, Phys. Rev. Phys. Educ. Res. 12, 010113 (2016).
[5] D. L. Reinholz, J. C. Corbo, M. H. Dancy, S. Deetz, and N. Finkelstein, in Transforming Institutions: $21^{\text {st }}$ Century Undergraduate STEM Education, edited by G. C. Weaver, A. L. Childress, and L. Slakey (Purdue University Press, West Lafayette, IN, 2015).

[6] J. C. Corbo, D. L. Reinholz, M. H. Dancy, and N. Finkelstein, in 2015 Physics Education Research Conference Proceedings (American Association of Physics Teachers, 2015). 